\title{
Histone Deacetylase Inhibitors (HDACi) Promote KLF5 Ubiquitination and Degradation in Basal-like Breast Cancer
}

\author{
Yanjie Kong1\#, Wenlong Ren²,8\#, Huan Fang2,3\#, Naseer Ali Shah2\#, Yujie Shi4, Dingyun You ${ }^{5}$, Chengang \\ Zhou ${ }^{2,3}$, Dewei Jiang2, Chuanyu Yang2, Huichun Liang2, Wenjin Liu' 2,3, Luzhen Wang, 2, Wenqiang Gan²,3, \\ Xing Wan', Fubing Li ${ }^{2,9 凶}$, Zhen Li ${ }^{1 凶}$, Ceshi Chen ${ }^{2 \bowtie}$, and Ni Xie ${ }^{1 \bowtie}$ \\ 1. Biobank, The First Affiliated Hospital of Shenzhen University, Shenzhen Second People's Hospital, Shenzhen, 518035, China \\ 2. Key Laboratory of Animal Models and Human Disease Mechanisms of Chinese Academy of Sciences and Yunnan Province, Kunming Institute of Zoology, \\ Kunming, Yunnan, China \\ 3. Kunming College of Life Sciences, University of Chinese Academy Sciences, Kunming, Yunnan, China \\ 4. Department of Pathology, Henan Provincial People's Hospital, Zhengzhou University, Zhengzhou, Henan, 450003, China \\ 5. Kunming Medical University, Kunming, Yunnan, 650500, China \\ 6. Department of Dermatology, Jingmen No.1 people's Hospital, Jingmen, Hubei, 448000, China \\ 7. Department of the Third Breast Surgery, the Third Affiliated Hospital of Kunming Medical University, Kunming, Yunnan, 650118, China \\ 8. School of Life Science, University of Science \& Technology of China. Hefei, Anhui, 230026, China \\ 9. Affiliated Cancer Hospital \& Institute of Guangzhou Medical University, Guangzhou, 510095, China \\ \#These authors contribute equally. \\ $\triangle$ Corresponding author: Ni Xie, E-mail: xn100@szu.edu.cn; Ceshi Chen, E-mail: chenc@mail.kiz.ac.cn; Zhen Li, Email: li_hazel@126.com; or Fubing Li, Email: \\ mtlfb0408@163.com
}

(0) The author(s). This is an open access article distributed under the terms of the Creative Commons Attribution License (https://creativecommons.org/licenses/by/4.0/). See http://ivyspring.com/terms for full terms and conditions.

Received: 2021.07.25; Accepted: 2022.02.09; Published: 2022.02.28

\begin{abstract}
Basal-like breast cancer (BLBC) accounts for approximately $15 \%$ of all breast cancer cases, and patients with BLBC have a low survival rate. Our previous study demonstrated that the KLF5 transcription factor promotes BLBC cell proliferation and tumor growth. In this study, we demonstrated that the histone deacetylase inhibitors (HDACi), suberoylanilide hydroxamic acid (SAHA), and trichostatin A (TSA), increased KLF5 acetylation at lysine 369 (K369), downregulated KLF5 protein expression levels, and decreased cell viability in BLBC cell lines. HDACi target KLF5 for proteasomal degradation by promoting KLF5 protein ubiquitination. K369 acetylation of KLF5 decreases the binding between KLF5 and its deubiquitinase, BAPI. These findings revealed a novel mechanism by which HDACi suppress BLBC, and a novel crosstalk between KLF5 protein acetylation and ubiquitination.
\end{abstract}

Key words: Histone deacetylase, HDAC1, trichostatin A, suberoylanilide hydroxamic acid, basal-like breast cancer, KLF5

\section{Introduction}

Globally, breast cancer is consistently the most common cancer type in women, as well as the leading cause of cancer death in this group [1]. Based on gene expression levels, breast cancer can be classified as luminal A, luminal B, HER2 positive, and basal-like subtypes [2]. Among these, basal-like breast cancers (BLBCs) are mostly ER $\alpha, \mathrm{PR}$, and HER2 triplenegative breast cancers (TNBCs), which comprise 15$20 \%$ of all breast cancer diagnoses and are associated with poor prognosis, high risk of distant recurrence, and low five-year survival [3]. Therefore, there is an urgent need to develop effective targeted therapies for BLBC.

Histone deacetylase (HDAC) inhibitors (HDACi) have emerged as a promising new class of multifunctional anticancer agents with the ability to suppress cancer cell migration, invasion, metastasis, and angiogenesis [4]. More than 15 HDACi have been tested in preclinical and early clinical studies [5]. Among these, vorinostat and panobinostat (LBH589; NVP-LBH589) have been approved by the FDA to treat cutaneous T-cell lymphoma and multiple 
myeloma, respectively [6]. We previously reported that trichostatin A (TSA) and suberoylanilide hydroxamic acid (SAHA) induce growth arrest and apoptosis in TNBC [7]. TSA selectively and consistently inhibited the proliferation of TNBC cell lines HCC1806 and HCC38 by inducing cell cycle arrest and apoptosis [8]. SAHA in combination with epigallocatechin-3-gallate suppressed breast cancer cell growth [9]. HDACi (SAHA or belinostat) reduced homologous recombination efficiency in TNBC, and sensitized TNBC to poly-ADP ribose polymerase (PARP) inhibition [10]. Panobinostat and salinomycin efficiently inhibited HCC1937 cell stemness, proliferation, survival, and migration [11]. Panobinostat inhibited epithelial-mesenchymal transition (EMT) and metastasis of MDA-MB-231 [12]. However, the mechanism by which TSA and SAHA suppress TNBC remains unclear.

The Krüppel-like factor 5 (KLF5) transcription factor is highly expressed in BLBC and induces a number of downstream target genes, such as CCND1 [13], FGF-BP1 [14], mPGES1 [15], TNFAIP2 [16], Slug [17], and IGFL1-AS1 and IGFL1 [18] which are associated with cell cycle, survival, migration, and stemness. Previous studies have shown that high expression levels of KLF5 are significantly associated with an increased risk of recurrence and poor prognosis in breast cancer patients [19]. KLF5 is a potential therapeutic target for BLBC [20]. KLF5 depletion suppresses BLBC initiation, growth, and metastasis [17, 21]. Furthermore, inhibition of KLF5 by mifepristone and its derivatives [22-24], metformin [25], mithramycin A [26], super-enhancer inhibitors [27], and the protein arginine N-methyltransferase 5 (PRMT5) inhibitor, PJ-68 [28], suppress BLBC stemness and tumor growth.

KLF5 can be acetylated [29]. In response to transforming growth factor $\beta$, the lysineacetyltransferase, p300, acetylates KLF5 at K369 to facilitate $p 15$ gene transcription and inhibit Myc gene transcription [30, 31]. KLF5 acetylation at K369 plays an important role in regulating prostate development and regeneration [32]. KLF5 can be deacetylated by HDAC1/2 and SET, which inhibits its function [33, 34]. HDAC4 promotes airway inflammation and remodeling by deacetylating KLF5, increasing KLF5 transcriptional activity, and facilitating the progression of asthma [35].

KLF5 is an unstable protein that undergoes ubiquitin-mediated proteasomal degradation [36]. E3 ligases WWP1 [37], Smurf2 [38], and SCF Fbw7 [39] have been shown to promote KLF5 ubiquitination and degradation. YAP and TAZ protect KLF5 from WWP1-mediated degradation [40, 41]. PRMT5 also stabilizes KLF5 by preventing KLF5 from SCFFbw7- mediated degradation [28]. Three deubiquitinases (DUBs), including BAP1 [21], ATXN3L [42], and USP3 [43] stabilize the KLF5 protein in BLBC.

Since both acetylation and ubiquitination occur at lysine residues, it is inevitable that there is crosstalk between two posttranslational modifications. It has been reported that acetylation could either block [44, $45]$ or promote $[46,47]$ ubiquitination. A recent study suggested that KLF5 acetylation may regulate KLF5 protein stability and that knockdown of HDAC1/2 in DU145 prostate cancer cells upregulated KLF5 protein acetylation and expression levels by blocking its degradation [34]. However, the mechanism by which KLF5 acetylation regulates its degradation is unclear.

In this study, we demonstrated that TSA and SAHA significantly downregulated the expression of KLF5 protein and suppressed the viability of BLBC cell lines. HDACi promoted the acetylation of KLF5 at K369, which decreased the association between BAP1 (a DUB) and KLF5. Thus, KLF5 acetylation promotes ubiquitination and proteasomal degradation in BLBC. Our findings shed light on the crosstalk between acetylation and ubiquitination of KLF5 and suggest that HDACi could be used to treat KLF5-positive BLBC.

\section{Materials and Methods}

\section{Cell lines, antibodies, and reagents}

MCF10A, HCC1937, HEK293T, and HCC1806 cell lines were purchased from the American Type Culture Collection (ATCC, Manassas, VA, USA). TSA, SAHA, MG132, and cycloheximide (CHX) were purchased from Sigma (St. Louis, MO, USA). Total acetylated KLF5 was detected by western blotting using an anti-acetylated lysine antibody (Minneapolis, MN. Cat\# AF3758); Ac-KLF5K369 (1:1000 in 5\% BSA) antibodies were generated in previous studies $[36,48]$. The anti-human FGF-BP1 (MAB1593) antibody was purchased from R\&D Systems (Minneapolis, MN, USA). Antibodies against HDAC1, acetylated lysine, Cyclin D1, p21, Flag, Ub, c-Myc, PARP, Caspase-3, Caspase-7, $\beta$-actin, and glyceraldehyde 3-phosphate dehydrogenase (GAPDH) were purchased from Cell Signaling Technology (Danvers, MA, USA). The siRNA target sequences for the human HDAC1 gene were HDAC1si\#1 (5'-GCGACTGTTTGAGAACCTT-3'), HDAC1 siRNA \#2 (5'-GGGATCGGTTAGGTTGCTT$\left.3^{\prime}\right)$, and HDAC1 si\#3 (5'-AGGCGGTGGTTACACCA TT-3').

\section{Stable KLF5, KLF5K369R, and KLF5K369Q expression}

Full-length wild-type human KLF5 was cloned 
into the pcDNA3.1 and pCDH vectors. The acetylation-deficient mutant KLF5 ${ }^{\mathrm{K} 369 \mathrm{R}}$ (KR) and the acetylation-mimicking mutant $\mathrm{KLF} 5 \mathrm{~K} 369 \mathrm{Q}$ (KQ) were generated by PCR-based mutagenesis, and confirmed by sequencing. Lentiviruses expressing KLF5 and its mutants were packaged and applied to infect MCF10A, HCC1937, and HCC1806 cells.

\section{Western blotting}

Western blotting was performed using a previously established procedure, and the KLF5 antibody was generated as described in our previous study [36]. After treatment, the cells were washed three times with $1 \times \mathrm{PBS}$ and lysed with lysis buffer for $30 \mathrm{~min}$. The cell lysate was centrifuged at 12,000 rpm for $10 \mathrm{~min}$ to collect the supernatant. Protein concentrations were detected using the Bradford assay (Bio-Rad Laboratories, Inc., Hercules, CA, USA). Equal amounts of proteins for each treatment were loaded and separated by SDS-PAGE, transferred to polyvinylidene fluoride membranes (GE Healthcare, Chicago, IL, USA), blocked with 5\% skim milk in $1 \times$ TBST for $1 \mathrm{~h}$ at room temperature, and subsequently incubated overnight at $4{ }^{\circ} \mathrm{C}$ with primary antibody. After washing three times with $1 \times$ TBST, the membrane was exposed to secondary peroxidase-conjugated antibody and visualized using western blotting detection reagents (GE Healthcare). GAPDH or $\beta$-actin was used as loading control.

\section{Cell viability measurement}

Cell proliferation in HCC1937 and HCC1806 cell lines was measured using a sulforhodamine B (SRB) assay (Sigma). Briefly, cells were seeded into 96-well plates at 5,000 cells/well. After $24 \mathrm{~h}$, the cells were treated with TSA or SAHA at the indicated concentrations, and equivalent DMSO served as a negative control. The cells were then fixed with $100 \mu \mathrm{L}$ of $10 \%$ trichloroacetic acid for $60 \mathrm{~min}$; subsequently they were washed five times with deionized water. The cells were stained with $100 \mu \mathrm{L}$ $0.4 \%(\mathrm{w} / \mathrm{v})$ SRB in $1 \%$ acetic acid for $5 \mathrm{~min}$, and then the plates were washed five times with $1 \%$ acetic acid, and dried. Finally, $100 \mu \mathrm{L}$ of $10 \mathrm{mM}$ Tris base was added to each well. Optical density at $540 \mathrm{~nm}$ was measured using a spectrophotometric plate reader. The cell viability values at different drug dosages were plotted in 2010 Excel (Microsoft, WA, USA); IC 50 values were obtained from the graphs.

\section{Ubiquitination assays}

Stable overexpression KLF5-3×Flag HCC1937 or HCC1806 cells were treated with TSA $(1 \mu \mathrm{M})$ or SAHA $(5 \mu \mathrm{M})$. After 2 days, the cells were treated with $20 \mu \mathrm{M}$ MG132 for $6 \mathrm{~h}$ to enrich polyubiquitinated
KLF5 proteins. The cells were harvested in lysis buffer (50 mM Tris-Cl and 1.5\% SDS; pH 6.8) using a six-well plate. Each well contained $150 \mu \mathrm{L}$ of lysis buffer. The cell lysate was boiled for $15 \mathrm{~min}$ to denature the proteins. BSA buffer (50 mM Tris-Cl, $180 \mathrm{mM} \mathrm{NaCl}$, $0.5 \%$ NP-40, and 0.5\% BSA; pH 6.8; $1.2 \mathrm{~mL}$ ) was added to dilute the samples. Flag-M2 beads $(30 \mu \mathrm{L}$ per sample; prewashed with BSA buffer three times) were added to immunoprecipitate Flag-KLF5 overnight with rotation in a cold room $\left(4^{\circ} \mathrm{C}\right)$. The beads were washed three times with $1 \mathrm{~mL}$ ice-cold BSA buffer, resuspended in $30 \mu \mathrm{L}$ of $1 \times$ SDS-PAGE sample buffer, boiled for $10 \mathrm{~min}$, and centrifuged for $2 \mathrm{~min}$ at 12,000 g. Ubiquitinated KLF5 was detected by Western blotting using the anti-Ub antibody.

\section{Immunohistochemical staining}

To confirm the correlation between KLF5 and HDAC1 in breast tumor tissues, the samples were fixed with $4 \%$ buffered formaldehyde for $48 \mathrm{~h}$ at room temperature and embedded in paraffin. Paraffinembedded clinical TNBC specimens were obtained from the First Affiliated Hospital, Zhengzhou University, Zhengzhou, China (Patient information is listed in Supplementary Table S1). Informed consent was obtained from all subjects. Two tissue microarrays containing 87 TNBC breast cancer tissues were constructed. For the immunohistochemistry (IHC) assay, the slides were deparaffinized, rehydrated, and pressure cooker heated for $2.5 \mathrm{~min}$ in EDTA for antigen retrieval. Endogenous peroxidase activity was inactivated by adding an endogenous peroxidase blocker (OriGene, Beijing, China) for 15 $\mathrm{min}$ at room temperature. Slides were incubated overnight at $4{ }^{\circ} \mathrm{C}$ with anti-KLF5 (1:1000) or anti-HDAC1 (1:2000). After $12 \mathrm{~h}$, the slides were washed three times with PBS and incubated with secondary antibodies (hypersensitive enzyme-labeled goat anti-mouse/rabbit IgG polymer (OriGene, Beijing, China) at room temperature for $20 \mathrm{~min}$, DAB concentrate chromogenic solution (1:200 dilution of concentrated DAB chromogenic solution), counterstained with $0.5 \%$ hematoxylin, dehydrated with graded concentrations of ethanol for 3 min each $(70 \%-80 \%-90 \%-100 \%)$, and finally stained with dimethyl benzene immunostained slides were evaluated by light microscopy. The IHC signal was scored using the 'Allred Score' method.

\section{Statistical analysis}

Data are shown as mean \pm standard deviation. The Student's t-test was used for statistical analysis. GraphPad Prism was used for all the statistical analyses. SPSS 20.0 software was used for Chi-square statistical analysis, The chi-square test was used to 
analyze the differences in HDAC1 and KLF5 expression among patients with different pathological grades. Fisher's exact probability test was used where the theoretical frequency was $<1$. McNemar's test was used to compare the expression consistency of HDAC1 and KLF5 in patients. A two-sided test with a significance level of $a=0.05$ was used for the statistical results. For the TNM stage comparison, we fit an ordinal variable of stage (I, II, and III) and estimated the relative risks (odds ratios) of one category increase in stage by triple-negative status. Repeated measures analysis of variance (RM-ANOVA) was applied to compare the cell viability of the time $(0,12,24,36$ and 48 hours after intervention) and the effect of time-group interaction. The normality of distributions was verified using the Shapiro-Wilk test. All reported P-values were two-sided. Statistical significance was set at ${ }^{*} \mathrm{P}<0.05$; $* * \mathrm{P}<0.01 ; * * * \mathrm{P}<0.001$

\section{Results}

TSA and SAHA suppressed BLBC cell growth, and promoted KLF5 acetylation, but downregulated the KLF5 protein levels

HDACi are known to be cytotoxic to TNBC [49].
We treated BLBC cell lines HCC1937 and HCC1806 with TSA and SAHA and found that the cell viability of both cell lines decreased in a dose-dependent manner. The $\mathrm{IC}_{50}$ values of SAHA in HCC1937 and HCC1806 were $8.3 \mu \mathrm{M}$ and $5.1 \mu \mathrm{M}$, respectively; the IC $_{50}$ values of TSA in HCC1937 and HCC1806 were 0.8 $\mu \mathrm{M}$ and $0.1 \mu \mathrm{M}$, respectively (Fig. 1A). Previous studies have shown that KLF5 promotes BLBC cell proliferation, survival, and migration [27] and that KLF5 is regulated by acetylation [50]. We wondered whether HDACi regulated KLF5 expression in BLBC. In contrast to the reported results in the DU145 prostate cancer cell line [34], both TSA and SAHA reduced KLF5 protein expression levels in HCC1937 and HCC1806 cells in a time-dependent manner (Fig. 1B). To investigate whether HDACi promoted the acetylation of KLF5, we performed Western blotting with an anti-KLF5 K369 acetylation-specific antibody and found that HDACi treatment increased KLF5 acetylation at K369 (Fig. 1B). Next, we investigated the mechanism by which HDACi downregulate KLF5 protein expression in BLBC. We demonstrated that KLF5 mRNA levels were not affected by SAHA or TSA treatment (Fig. S1A-B). Thus, it is possible that HDACi promote KLF5 protein degradation.
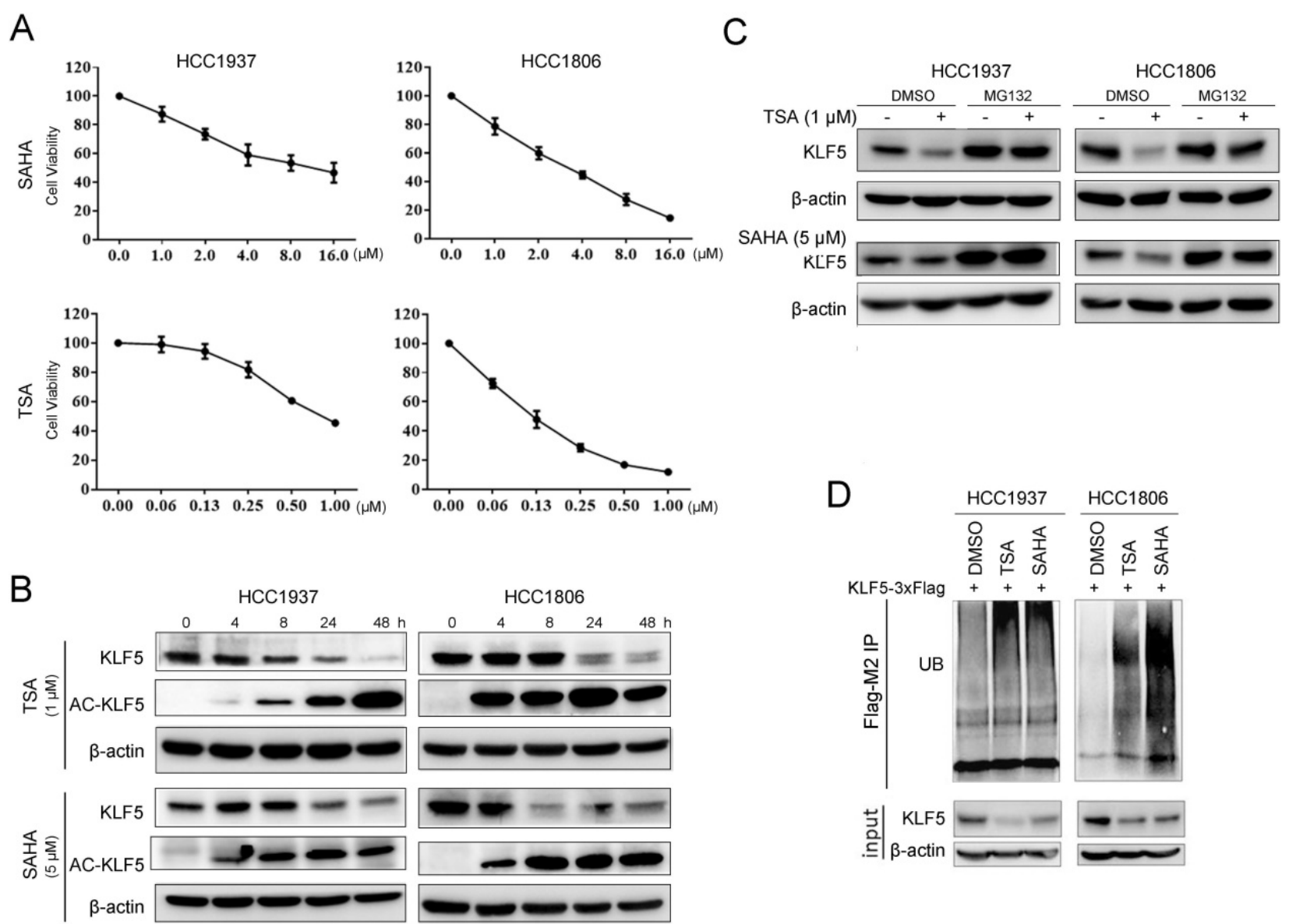

Figure 1. HDACi inhibited KLF5 expression and cell viability in BLBC cell lines. A, TSA and SAHA inhibited the HCC1806 and HCC1937 cell viability in a dose-dependent manner. TSA and SAHA were added to media at the indicated concentration, and cell viability was assessed after $48 \mathrm{~h}$ by sulforhodamine $\mathrm{B}$ (SRB) assays. B, TSA 
and SAHA decreased KLF5 protein expression in a time-dependent manner. HCC1937 and HCC1806 cells were treated with TSA (1 $\mu \mathrm{M})$ and SAHA $(5 \mu \mathrm{M})$ for different time periods $(0,4,8,24,48 \mathrm{~h})$, and KLF5 protein expression was analyzed by western blotting. $C$, the proteasome inhibitor MG 132 blocked HDACi-induced KLF5 decrease in $\mathrm{HCCl} 806$ and $\mathrm{HCC} 1937$ cell lines. The cells were treated with TSA $(1 \mu \mathrm{M})$ and SAHA $(5 \mu \mathrm{M})$ for $8 \mathrm{~h}$ and then MG $132(3 \mu \mathrm{M})$ were added for $8 \mathrm{~h}$. Cell lysates were collected for western blotting. D, these cells were treated with TSA $(1 \mu \mathrm{M})$ and SAHA $(5 \mu \mathrm{M})$ for $8 \mathrm{~h}$ and then MG132 $(3 \mu \mathrm{M})$ were added for the next $8 \mathrm{~h}$. Cell lysates were collected for western blotting.

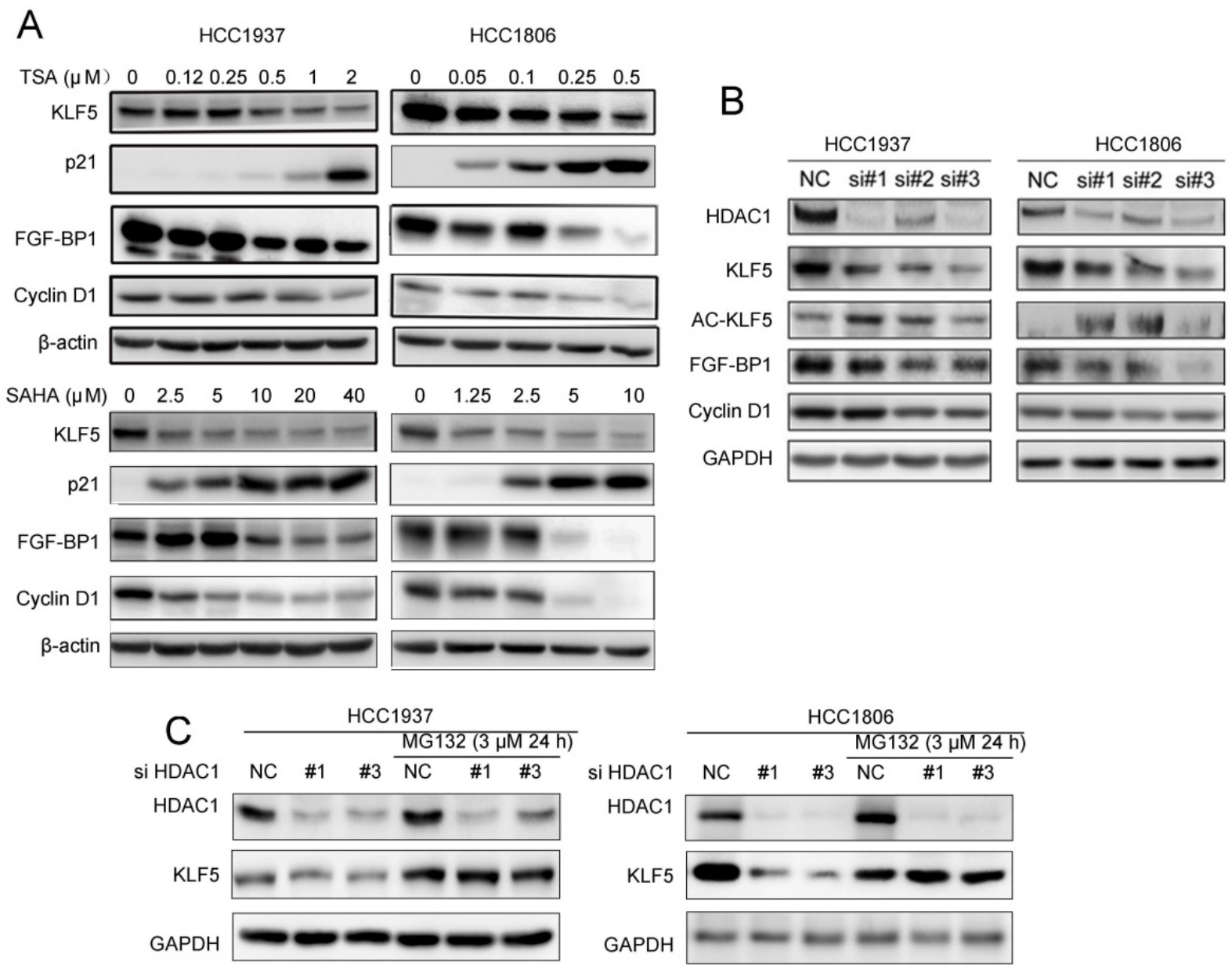

Figure 2. Both HDACi and HDACI knockdown downregulated the expression of KLF5 protein and its downstream targets genes. A, HDACi inhibited KLF5-mediated downstream target gene (FGF-BPI, Cyclin DI, and p21 expression changes. HCC1937 and HCC1806 cells were treated with HDACi for $48 \mathrm{~h}$ at the indicated concentrations. The protein expression was measured by Western blotting. B, HDACl knockdown by three different siRNAs in $\mathrm{HCCl} 1937$ and $\mathrm{HCCl} 1806$ cells for $48 \mathrm{~h}$; cell lysates were collected for western blotting, which indicated that KLF5 and its downstream target gene (FGF-BPI and Cyclin DI) expression were blocked. C, HDACI knockdown-induced reduction in KLF5 expression in BLBC was inhibited by proteasome inhibitor. HDACl was knocked down by three different siRNAs in HCC1937 and $\mathrm{HCCl} 1806$ cells for $24 \mathrm{~h}$. Subsequently, MG132 $(3 \mu \mathrm{M})$ was used to treat the cells for $24 \mathrm{~h}$. Cell lysates were collected for Western blotting.

In previous reports, KLF5 was degraded by proteasomes [39, 51]. When we treated BLBC cells with the proteasome inhibitor MG132, the HDACi-induced decrease in KLF5 levels was blocked (Fig. 1C). Furthermore, we showed that TSA and SAHA increased KLF5 ubiquitination (Fig. 1D).

\section{TSA and SAHA downregulated KLF5 and downstream target gene expression by inhibiting HDACI}

Since HDACi down-regulate KLF5 expression in BLBC, we wondered whether HDACi suppress KLF5 transcriptional activity towards its downstream target genes. We treated HCC1937 and HCC1806 cells with TSA and SAHA, and measured the protein levels of KLF5 downstream target genes, including p21, FGF-BP1, and Cyclin D1. As expected, HDACi decreased the expression levels of FGF-BP1 and Cyclin D1, and increased the expression levels of p21 (Fig. 2A).

Both TSA and SAHA are inhibitors of class I deacetylases, and HDAC1 was reportedly responsible for KLF5 deacetylation in a previous study [50]. We silenced HDAC1 with three different siRNAs and measured the protein levels of KLF5 and its downstream target genes. As shown in Fig. 2B, HDAC1 knockdown increased KLF5 acetylation and decreased the protein levels of KLF5 and its target genes, including FGF-BP1 and CCND1, in both BLBC cell lines. When we added MG132 to HDAC1 knockdown groups, the decrease in KLF5 protein levels was inhibited (Fig. 2C). Furthermore, KLF5 mRNA levels were not decreased by HDAC1 knockdown (Fig. S1C). 


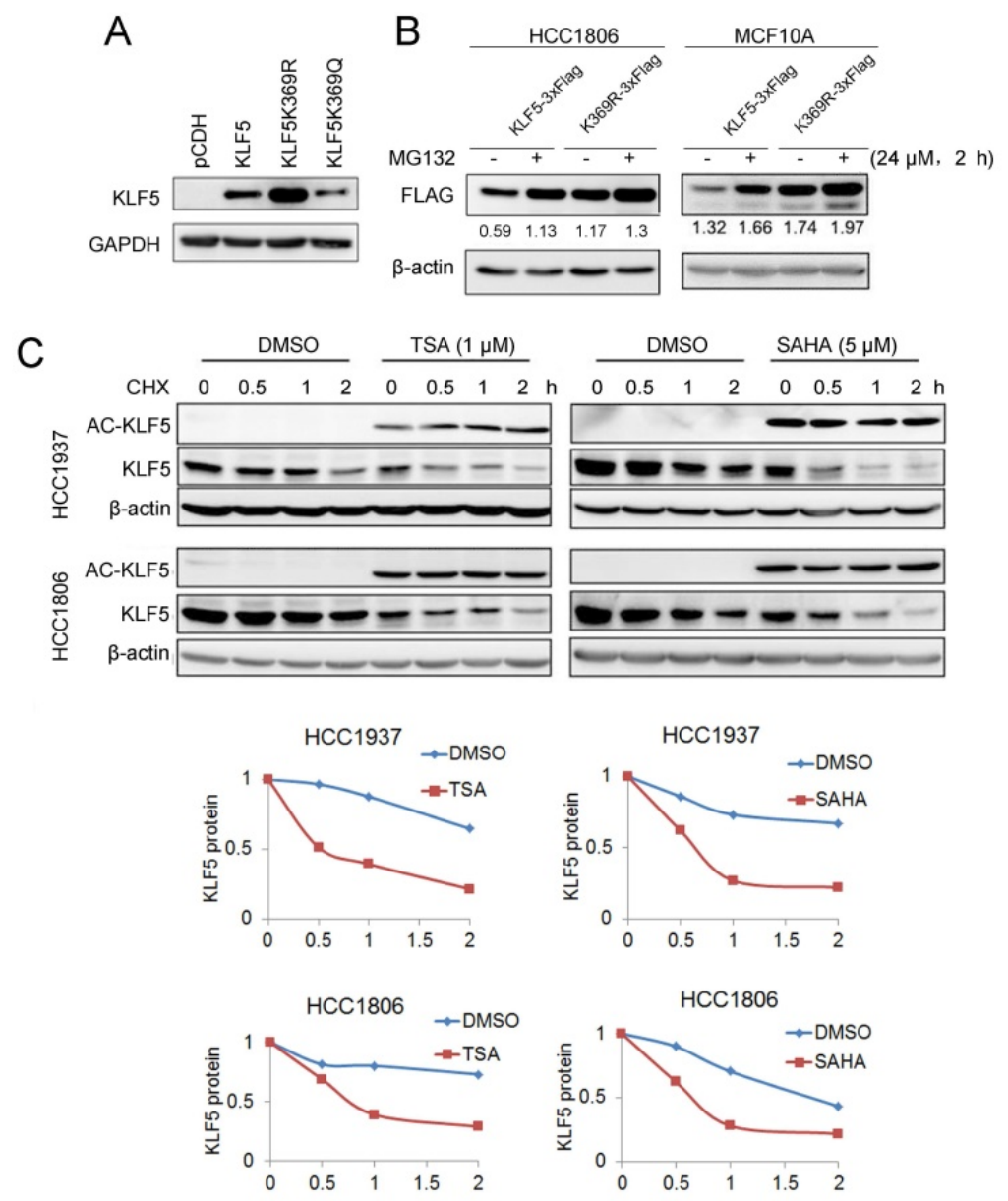


mutant is BAP1C91S) [21], USP3 (enzyme activity mutant is USP3C186S) [43], ATXN3L [42], YAP (interaction with KLF5 mutant is YAP W1W2) [41], and TAZ (interaction with KLF5 mutant is TAZ $\Delta W W$ ) [40]. To characterize the mechanism by which K369 acetylation destabilizes KLF5, we first tested whether KLF5 E3 ligases could promote K369R mutant protein degradation. Three KLF5 E3 ubiquitin ligases (WWP1, Fbw7, and Smurf2) efficiently decreased KLF5 KR protein levels (Fig. S2).

Next, we tested whether KLF5 DUBs or YAP/TAZ could protect the KQ protein from degradation. We co-transfected BAP1, USP3, ATXN3L, YAP, and TAZ with WT or KQ mutant KLF5 into HEK293T cells and detected KLF5 protein levels. We demonstrated that only BAP1 could not efficiently protect KLF5 ${ }^{\mathrm{K} 369} \mathrm{Q}$ from degradation (Fig. $4 \mathrm{~A})$.

To investigate whether acetylated KLF5 protein escaped from BAP1-mediated stabilization, we performed CHX assays to measure the KLF5 protein half-lives. In agreement with our previous report [21], BAP1 efficiently extended the half-life of the WT KLF5 protein; however, BAP1 failed to extend the half-life of KLF5 ${ }^{\mathrm{K} 369 \mathrm{Q}}$ (Fig. 4B).

We suspected that the acetylated KLF5 protein may decrease the interaction with BAP1. Indeed, BAP1 efficiently immunoprecipitated WT KLF5 protein, but not KLF5 ${ }^{\mathrm{K} 369 \mathrm{Q}}$ (Fig. 4C). Consistently, KLF5 ${ }^{\mathrm{K} 369 \mathrm{Q}}$ did not efficiently immunoprecipitate BAP1 (Fig. 4D). These results suggest that BAP1 does not efficiently interact with K369 acetylated KLF5; therefore, it does not protect it from degradation.

\section{KLF5 expression levels positively correlated with HDAC1 in TNBC tissues}

Given that KLF5 directly interacts with HDAC1 [53, 54], HDAC1 knockdown decreased KLF5 protein levels in BLBC cell lines (Fig. 2C). We wondered whether the expression levels of KLF5 and HDAC1 in TNBC tissues were positively correlated.

Clinic pathological parameters, including patient age, tumor size, lymph node status at the time of diagnosis, and follow-up status, including adjuvant treatment and tumor recurrence, were retrospectively obtained from the Department of Pathology, Henan Provincial People's Hospital, Zhengzhou University, Henan, China. We performed IHC analyses on two breast cancer tissue chips containing a total of 87 patients with TNBC (Table S1), showing that tumors with high KLF5 protein expression levels were significantly correlated with HDAC1 expression. McNemar's consistency test showed that the consistency of HDAC1 and KLF5 expression in patients with invasive ductal carcinoma was statistically significant (kappa $=0.287, \quad \mathrm{P}<0.001)$. Spearman rank correlation analysis showed that HDAC1 and KLF5 expression were positively correlated $(r=0.379, \mathrm{P}<0.001)$ (Fig. 5 and Table 1$)$.

Table 1. Consistency and correlation of KLF5 expression among different $\mathrm{HDACl}$ expression groups

\begin{tabular}{lllllll}
\hline KLF5 & HDAC1 & Kappa & $\begin{array}{l}\text { P } \\
\text { (McNemar) }\end{array}$ & $\begin{array}{l}\mathrm{P} \\
\text { (Spearman) }\end{array}$ \\
\cline { 2 - 3 } & Negative & Positive & & $<0.001$ & $0.379<0.001$ \\
\hline Negative & $8(9.1 \%)$ & $23(26.1 \%)$ & 0.287 & $<0.01$ & \\
Positive & $1(1.1 \%)$ & $56(63.6 \%)$ & & & & \\
\hline
\end{tabular}

\section{HDACi and BRD4i additively inhibit the KLF5 expression and BLBC cell growth.}

SAHA has been tested for TNBC patients in clinical trials in combination with chemotherapy. Since SAHA inhibits the KLF5 expression, we combined SAHA and BRD4 inhibitor 870 which was reported to inhibit KLF5 transcription in BLBC [27]. We found that these two compounds additively decreased the expression of KLF5 and its target genes, including FGF-BP1 and CyclinD1, increased the expression of p21, decreased cell viability of BLBC cells (Fig. 6A).

To test whether HDACi function through KLF5, we knocked down KLF5 in HCC1937 and HCC1806 cells and treated the cells with HDACi. As shown in Fig. 6B, KLF5 depletion significantly decreased the efficacies of HDACi. These results suggest that HDACi inhibit BLBC through targeting KLF5, at least in part. As expected, HDACi inhibit BLBC also through other mechanisms in the absence of KLF5.

\section{Discussion}

In this study, we demonstrated that HDAC inhibitors, TSA and SAHA, destabilized KLF5 protein and suppressed BLBC viability. We also showed that HDAC1 stabilized the KLF5 protein in BLBC cell lines. KLF5 acetylation at K369 promoted its ubiquitination and degradation because of its association with BAP1. These findings suggest that HDACi may be used to treat HDAC1-and KLF5-positive BLBC in the future.

Our conclusion is contrary to a previous report that knockdown of HDAC1/2 in the DU145 prostate cancer cell line promotes KLF5 acetylation at K369 but protects KLF5 from degradation [50]. This could be attributed to the different cell types. We showed that KLF5 acetylation at K369 promoted ubiquitination. Most recently, it was reported that the growth of KLF5-K369R overexpression prostate cancer cells in nude mice is faster than WT KLF5 overexpression cells. Simultaneously, the growth of KLF5-K369Q overexpression cells is slower than WT KLF5 
overexpression cells [55]. These results support our hypothesis that KLF5-K369R is more stable and oncogenic in vivo. We found that KLF5 ${ }^{\mathrm{K} 369 \mathrm{Q}}$ decreased its interaction with BAP1, a KLF5 DUB [21]. We previously found that the KLF5 C-terminus (373-457) interacted with BAP1 [21]. Since K369 is very close to this region, it is reasonable that KLF5 acetylation at
K369 could decrease its interaction with BAP1, although the exact mechanism requires further investigation. Previous studies reported that acetylation promoted protein degradation in hypoxia-inducible factor 1-alpha (HIF-1a) [46], GATA1 [56], and retinoblastoma $(\mathrm{Rb})$ proteins [47].
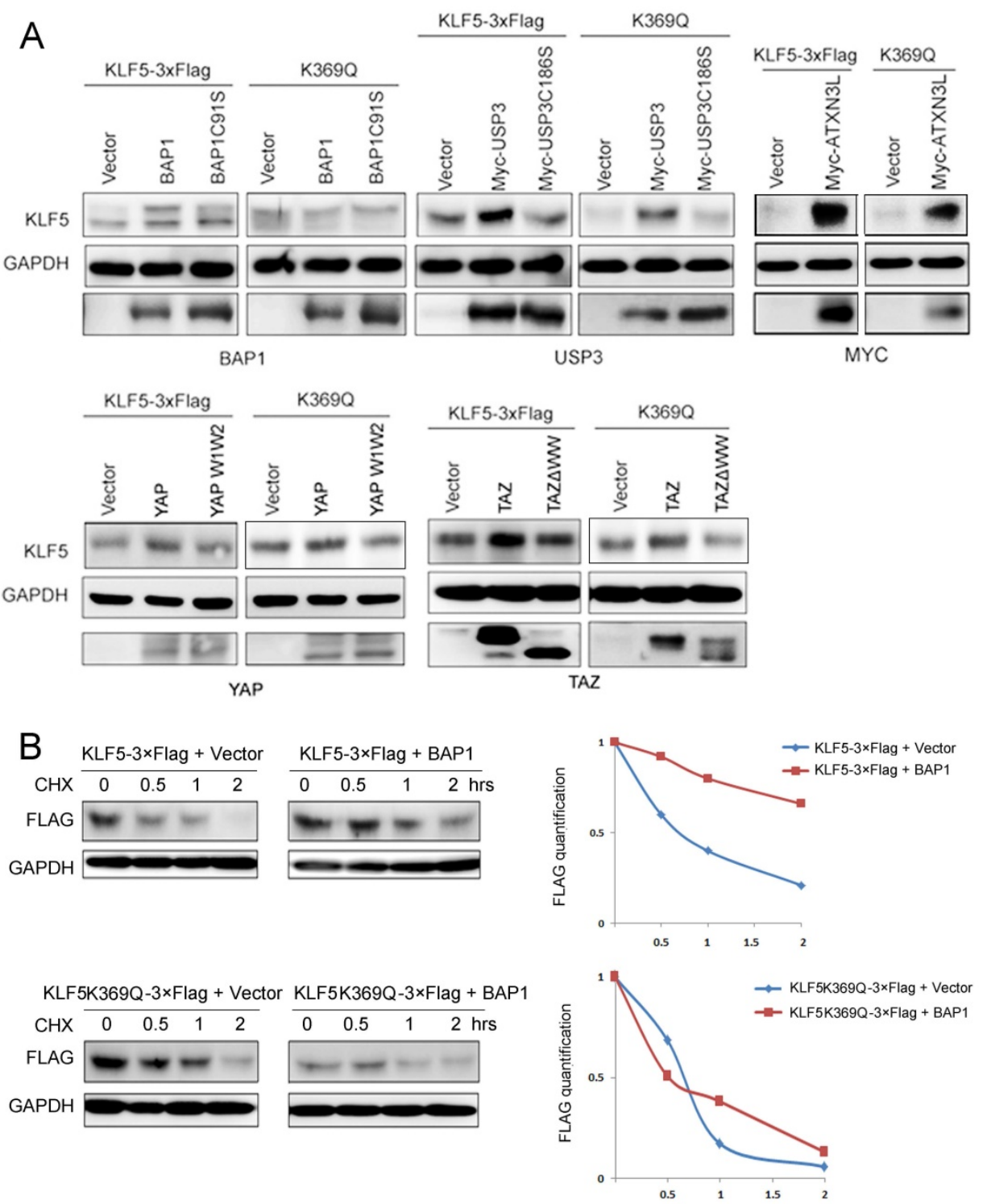

C

D

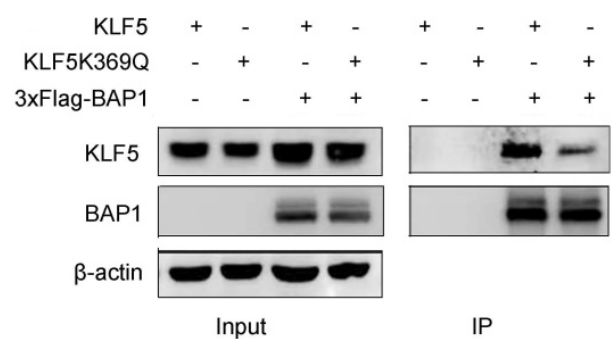

$3 \times$ Flag-KLF5 +-+-

3XFlag-KLF5K369Q - + - + +

BAP1 -

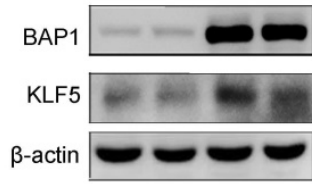

Input

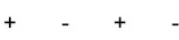

$-\quad+-+$

$-+++$

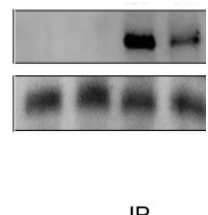

Figure 4. KLF5 acetylation at K369 suppressed its interaction with BAP1. A, BAP1 did not stabilize KLF5-K369Q protein. KLF5 and KLF5-K369Q were co-transfected with known KLF5 protein stabilizers (BAPI, USP3, ATXN3L, YAP, and TAZ) in HEK293T cells. Protein expression levels were detected by western blotting. B, BAP1 extended the protein half-life of WT KLF5, but not KLF5-K369Q. KLF5-3×Flag and KLF5-K369Q-3×Flag were co-transfected with empty vector and BAP1 in HEK293T cells. The cells were 
treated with $\mathrm{CHX}(50 \mu \mathrm{g} / \mathrm{mL})$ for $0.5,1$, and $2 \mathrm{~h}$. The quantitative results were plotted on the right. C, $\mathrm{K} 369 \mathrm{Q}$ mutation decreased the protein interaction between $\mathrm{KLF} 5$ and BAPI. KLF5, KLF5-K369Q, and 3×Flag-BAPI were co-expressed in HEK293T cells. 3×Flag-BAPI was immunoprecipitated with anti-Flag-M2 beads. Protein expression levels were detected by Western blotting. D, K369Q mutation decreased the protein interaction between KLF5 and BAPI. KLF5-3×Flag, KLF5-K369Q-3×Flag, and BAPI were co-expressed in HEK293T cells. KLF5-3×Flag and KLF5-K369Q-3×Flag proteins were immunoprecipitated with anti-Flag-M2 beads. Protein expression levels were detected by Western blotting.

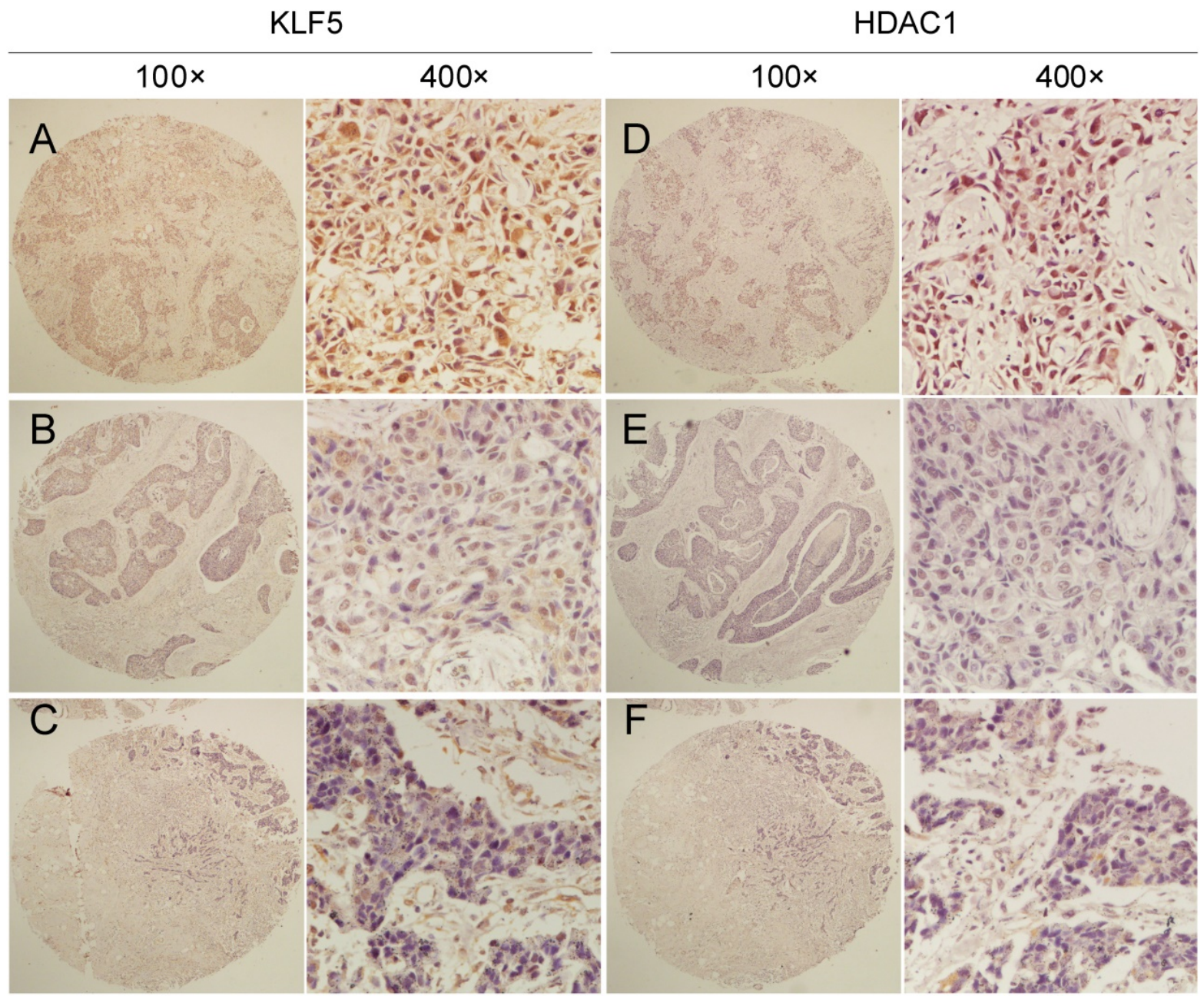

Figure 5. The protein expression of HDACI and KLF5 were positively correlated in TNBC specimens. Representative IHC images of KLF5 and HDACl protein expression in breast cancer tissues are shown. The final expression assessment was performed by combining the two scores $(0-2=l o w, 3-5=$ intermediate, $6-7=$ high), $A$ and $D$ indicate high scores, $B$ and $E$ indicate intermediate scores, and $C$ and $F$ indicate low scores.

It is well known that TNBC are heterogeneous. TNBC was suggested to be classified into four different subtypes [57]. KLF5 is highly expressed in basal-like breast cancers, including HCC1937 and HCC1806 cell lines, in contrast, KLF5 is lowly expressed in MDA-MB-231 cell line [20]. HDACi also decreased the KLF5 protein expression levels in two different BLBC cell lines, such as BT549 and SUM149PT (Fig. S3), suggesting that inhibition of KLF5 by HDACi is a common phenomenon in BLBC.

It has been reported that KLF5 is positively regulated by the acetylase p300/CBP [54]. GCN5 forms a complex with KLF5 and promotes KLF5 acetylation to facilitate lung cancer cell proliferation [58]. Several deacetylases, including SET and HDAC1-4, have been reported to deacetylate KLF5 $[54,59]$. In particular, HDAC1 can negatively regulate
KLF5 through direct interaction, and inhibit the DNA-binding activity of KLF5 [50, 54]. Recently, HDAC3 was shown to interact with KLF5 to promote prostate cancer autophagy in response to docetaxel treatment [60]. We tested whether the HDAC family (HDAC1-11, SIRT1-7) and HAT family (p300/CBP, GCN5, PCAF, and Tip60) could regulate KLF5 protein expression levels, and found that only HDAC1 stabilized KLF5 in BLBC (data not shown and Fig. 2).

Several HDACi are FDA-approved for the treatment of patients with hematological malignancies, e.g., romidepsin, vorinostat, and belinostat for T-cell lymphomas, and panobinostat for myeloma. In a phase III trial, postmenopausal women with HR+HER2- advanced-stage breast cancer, resistant to endocrine therapy, benefitted from tucidinostat, a new HDACi [61]. In clinical trials, 
A

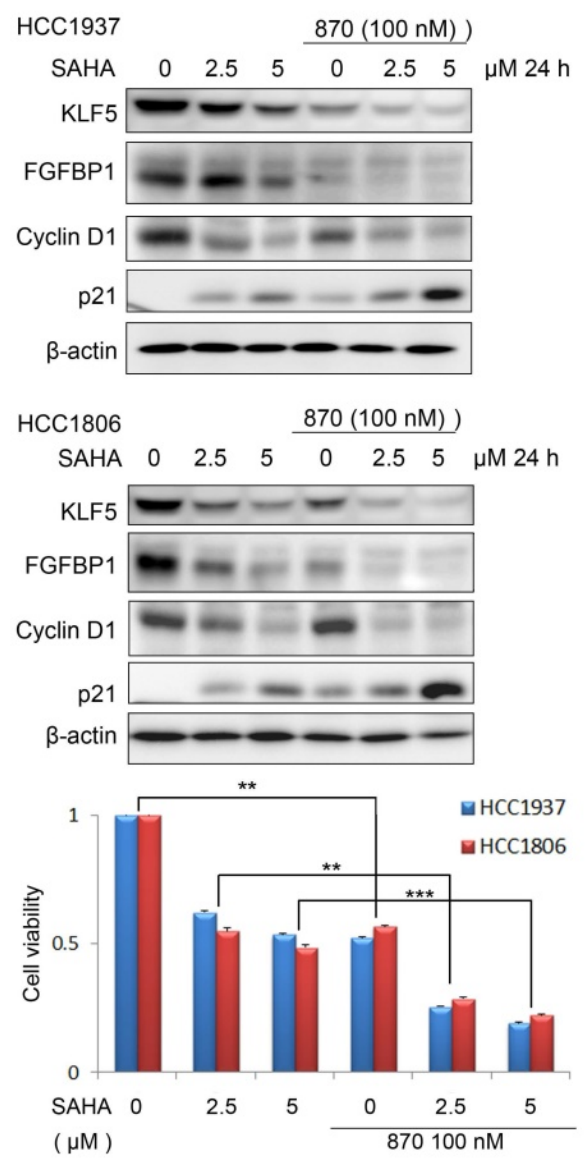

B

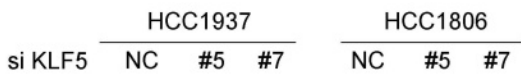

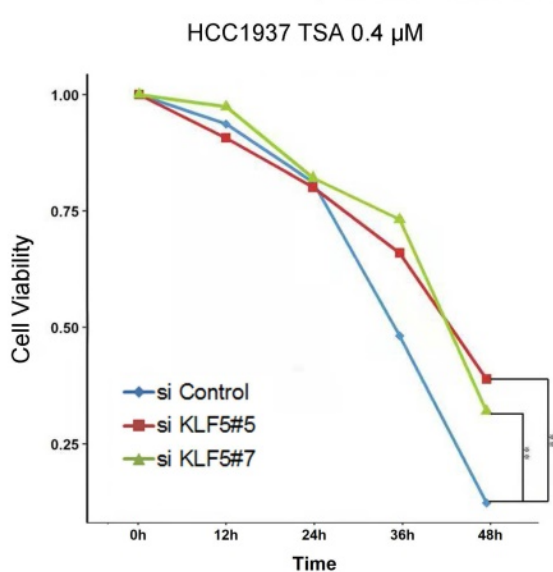

HCC1937 SAHA $3.5 \mu \mathrm{M}$

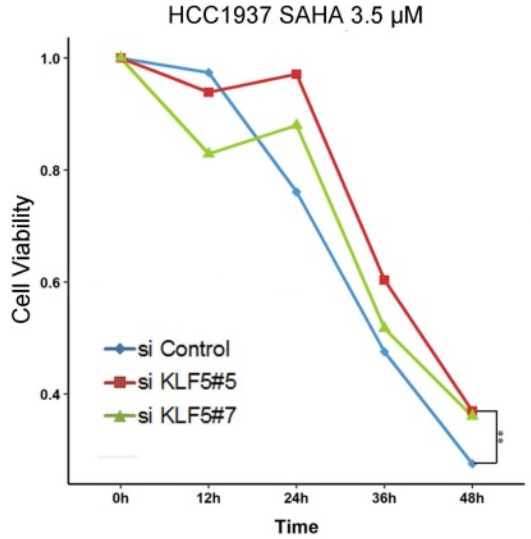

HCC1806 TSA $0.5 \mu \mathrm{M}$
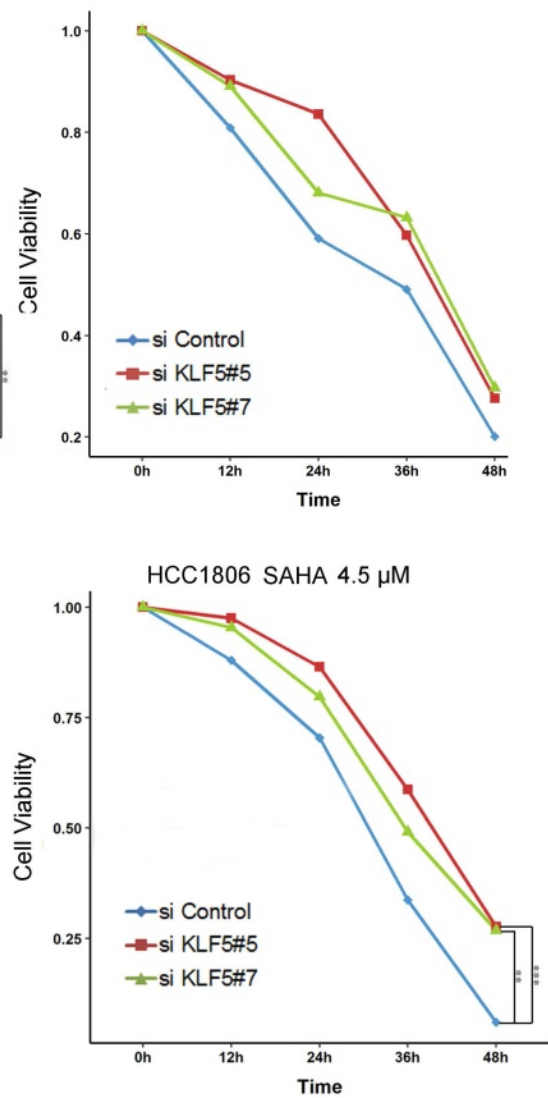

Figure 6. HDACi in combination with BRD4i inhibit the KLF5 expression and function and the survival of BLBC cells. A, HDACi were combined with compound 870 to regulate the expression of KLF5 and its downstream target genes (FGF-BP1, Cyclin D1, and p21). HCC1937 and HCC1806 cells were treated with HDACi and compound 870 for $24 \mathrm{~h}$ at the indicated concentrations. The protein expression was measured by Western blotting, and cell viability was assessed after $24 \mathrm{~h}$ by the SRB assay. B, KLF5 knockdown decreased the cytotoxicity of HDACi in BLBC cell lines. KLF5 was knocked down by two different siRNAs in HCC1937 and HCC1806 cells and cell lysates were collected for Western blotting. The cells were treated with HDACi for $48 \mathrm{~h}$ to measure cell viability by the SRB assay.

SAHA was tested in combination with tamoxifen, olaparib, capecitabine (xeloda), paclitaxel, and bevacizumab against metastatic breast cancer [62]. Carboplatin and nab-paclitaxel, with or without SAHA, have been tested for the treatment of women with newly diagnosed, operable breast cancer [63, 64].

HDACi suppress BLBC through KLF5, at least in part. After we depleted KLF5 in HCC1937 and HCC1806 cells, the inhibitory effects of HDACi were compromised (Fig. 6B). However, when we stably overexpressed KLF5 in HCC1937, HCC1806 and MCF10A, we could not rescue the loss of cell viability and apoptosis induced by HDACi (data not shown). We could not understand the mechanism at present. We observed that HDACi also decreased the c-Myc protein levels in these breast cell lines, suggesting that HDAC inhibitors may function through multiple targets besides KLF5.

In conclusion, TSA and SAHA promoted KLF5 protein degradation in BLBC by inhibiting HDAC1 activity and increasing KLF5 acetylation at K369, which abrogated its interaction with BAP1, a KLF5 DUB. Therefore, HDACi may hold potential for further development of anti-tumor regimens for BLBC.

\section{Abbreviations}

BLBC: basal-like breast cancer; CHX: cycloheximide; DUB: deubiquitinase; GAPDH: glyceraldehyde 3-phosphate dehydrogenase; HDAC: histone deacetylase; HDACi: histone deacetylase inhibitors; IHC: immunohistochemistry; KLF5: Krüppel-like factor 5; KQ: acetylation-mimicking mutant KLF5 K369Q; KR: acetylation-deficient mutant KLF5 ${ }^{K 369 R}$; PARP: poly-ADP ribose polymerase; SAHA: suberoylanilide hydroxamic acid; SRB: sulforhodamine $\mathrm{B}$; TNBC: triple-negative breast cancer; TSA: trichostatin A; WT: wild-type. 


\section{Supplementary Material}

Supplementary figures and table.

https://www.ijbs.com/v18p2104s1.pdf

\section{Acknowledgments}

We sincerely thank Dr. Jin-Tang Dong for proving the KLF5 ${ }^{K 369 R}$ plasmid, Dr. Linqiang Zhang for proving the HDAC1-11 plasmids, and Dr. Tiebang Kang for proving the Sirt1 and 2 plasmids. This study was supported in part by grants National Key Research and Development Program of China (2020YFA0112300 and 2018YFC2000400 to Chen, C.). The National Nature Science Foundation of China (81972003 and 82172356 to Xie, N., U2102203 and 81830087 to Chen, C., 82060481 to Li, Z., 81402206 , 81773149, and U1702283 to Kong, Y., and 82000817 to Li, F.). The National Postdoctoral Program for Innovative Talents (BX20190088 to Li, F.). The Natural Science Foundation of Guangdong (2017A030313668 and 2021A1515012144), Sanming Project of Medicine in Shenzhen (SZSM201612031), Shenzhen Municipal Government of China (JCYJ20170817171808368, JCYJ20170818085657917, JCYJ20180507184647104, KQTD20170810160226082) to Xie, N. Shenzhen Municipal Government of China (JCYJ20210324103 603011 to Kong, Y.), The Yunnan Applied Basic Research Projects (202101AS070050 to Chen, C, 202101AT070024 and 2019FE001-309 to Li, Z, and 2018FB134 to Kong, Y.), Chinese Postdoctoral Science Foundation (182703 and 230794 to Kong, Y. and 2019 M662869 to Li, F.), and CAS "Light of West China" Program (Kong, Y.).

\section{Author Contributions}

FL, ZL, CC, and NX designed the experiments and provided financial support. YK, WR, HF, and NS carried out most experiments and analyzed data. DY did the statistical analysis. $\mathrm{CY}$ performed the IHC and HE staining. YS provided clinical samples. CZ, LW, and XW helped with the experiments. YK and CC wrote the manuscript.

\section{Competing Interests}

The authors have declared that no competing interest exists.

\section{References}

1. Jemal A, Bray F, Center MM, Ferlay J, Ward E, Forman D. Global cancer statistics. CA: A Cancer Journal for Clinicians. 2011; 61: 69-90.

2. Nielsen TO, Hsu FD, Jensen $K$, Cheang M, Karaca G, Hu Z. Immunohistochemical and clinical characterization of the basal-like subtype of invasive breast carcinoma. Clinical Cancer Research. 2004; 10: 5367-74.

3. Tan AR. Triple-Negative Breast Cancer: Springer International Publishing; 2017.

4. Liu T, Kuljaca S, Tee A, Marshall GM. Histone deacetylase inhibitors: Multifunctional anticancer agents. Cancer Treatment Reviews. 2006; 32: 157-65.

5. Lane AA, Chabner BA. Histone Deacetylase Inhibitors in Cancer Therapy. Journal of Clinical Oncology. 2009; 27: 5459-68.
6. Zucchetti B, Shimada AK, Katz A, Curigliano G. The role of histone deacetylase inhibitors in metastatic breast cancer. Breast. 2019; 43: 130-4.

7. Fang H, Du G, Wu Q, Liu R, Chen C, Feng J. HDAC inhibitors induce proline dehydrogenase (POX) transcription and anti-apoptotic autophagy in triple negative breast cancer. Acta Biochim Biophys Sin (Shanghai). 2019; 51: 1064-70.

8. Song X, Wu JQ, Yu XF, Yang XS, Yang Y. Trichostatin A inhibits proliferation of triple negative breast cancer cells by inducing cell cycle arrest and apoptosis. Neoplasma. 2018; 65: 898-906.

9. Lewis KA, Jordan HR, Tollefsbol TO. Effects of SAHA and EGCG on Growth Potentiation of Triple-Negative Breast Cancer Cells. Cancers. 2019; 11.

10. Marijon H, Lee DH, Ding L, Sun H, Gery S, de Gramont A, et al. Co-targeting poly(ADP-ribose) polymerase (PARP) and histone deacetylase (HDAC) in triple-negative breast cancer: Higher synergism in BRCA mutated cells. Biomedicine \& Pharmacotherapy. 2018; 99: 543-51.

11. Kai M, Kanaya N, Wu SV, Mendez C, Nguyen D, Luu T, et al. Targeting breast cancer stem cells in triple-negative breast cancer using a combination of LBH589 and salinomycin. Breast Cancer Res Treat. 2015; 151: 281-94.

12. Rhodes LV, Tate CR, Segar HC, Burks HE, Phamduy TB, Hoang V, et al. Suppression of triple-negative breast cancer metastasis by pan-DAC inhibitor panobinostat via inhibition of ZEB family of EMT master regulators. Breast Cancer Res Treat. 2014; 145: 593-604

13. Chen C, Benjamin MS, Sun X, Otto KB, Guo P, Dong XY, et al. KLF5 promotes cell proliferation and tumorigenesis through gene regulation and the TSU-Pr1 human bladder cancer cell line. International journal of cancer. 2006; 118: 1346-55.

14. Zheng HQ, Zhou Z, Huang J, Chaudhury L, Dong JT, Chen C. Kruppel-like factor 5 promotes breast cell proliferation partially through upregulating the transcription of fibroblast growth factor binding protein 1. Oncogene. 2009; 28 : 3702-13.

15. Xia H, Wang C, Chen W, Zhang H, Chaudhury L, Zhou Z, et al. Kruppel-like factor 5 transcription factor promotes microsomal prostaglandin E2 synthase 1 gene transcription in breast cancer. Journal of Biological Chemistry. 2013; 288: 26731-40.

16. Jia L, Zhou Z, Liang H, Wu J, Shi P, Li F, et al. KLF5 promotes breast cancer proliferation, migration and invasion in part by upregulating the transcription of TNFAIP2. Oncogene. 2016; 35: 2040-51.

17. Liu R, Shi $\mathrm{P}$, Zhou Z, Zhang H, Li W, Zhang H, et al. Krüpple-like factor 5 is essential for mammary gland development and tumorigenesis. The Journal of pathology. 2018; 246: 497-507.

18. Wang $\mathrm{H}$, Shi $\mathrm{Y}$, Chen $\mathrm{CH}$, Wen $\mathrm{Y}$, Zhou Z, Yang $\mathrm{C}$, et al. KLF5-induced IncRNA IGFL2-AS1 promotes basal-like breast cancer cell growth and survival by upregulating the expression of IGFL1. Cancer Lett. 2021; 515: 49-62.

19. Takagi K, Miki Y, Onodera Y, Nakamura Y, Ishida T. Krüppel-like factor 5 in human breast carcinoma: a potent prognostic factor induced by androgens. Endocr Relat Cancer. 2012; 19: 741-50.

20. Luo $\mathrm{Y}, \mathrm{Chen} \mathrm{C}$. The roles and regulation of the KLF5 transcription factor in cancers. Cancer Sci. 2021.

21. Qin J, Zhou Z, Chen W, Wang C, Zhang H, Ge G, et al. BAP1 promotes breast cancer cell proliferation and metastasis by deubiquitinating KLF5. Nat Commun. 2015; 6: 8471.

22. Liu R, Shi P, Nie Z, Liang H, Zhou Z, Chen W, et al. Mifepristone Suppresses Basal Triple-Negative Breast Cancer Stem Cells by Down-regulating KLF5 Expression. Theranostics. 2016; 6: 533-44.

23. Lin Y, Liu R, Zhao P, Ye J, Zheng Z, Huang J, et al. Discovery of novel mifepristone derivatives via suppressing KLF5 expression for the treatment of triple-negative breast cancer. Eur J Med Chem. 2018; 146: 354-67.

24. Liu $\mathrm{R}$, Chen $\mathrm{H}$, Zhao $\mathrm{P}$, Chen $\mathrm{CH}$, Liang $\mathrm{H}$, Yang $\mathrm{C}$, et al. Mifepristone Derivative FZU-00,003 Suppresses Triple-negative Breast Cancer Cell Growth partially via miR-153-KLF5 axis. International journal of biological sciences. 2020; 16: 611-9.

25. Shi P, Liu W, Tala, Wang H, Li F, Zhang H, et al. Metformin suppresses triple-negative breast cancer stem cells by targeting KLF5 for degradation. Cell Discov. 2017; 3: 17010.

26. Liu R, Zhi X, Zhou Z, Zhang $H$, Yang R, Zou $T$, et al. Mithramycin A suppresses basal triple-negative breast cancer cell survival partially via down-regulating Kruppel-like factor 5 transcription by Sp1. Scientific reports. 2018; 8: 1138.

27. Chen $\mathrm{CH}$, Yang N, Zhang Y, Ding J, Zhang W, Liu R, et al. Inhibition of super enhancer downregulates the expression of KLF5 in basal-like breast cancers. Int J Biol Sci. 2019; 15: 1733-42.

28. Wang X, Qiu T, Wu Y, Yang C, Li Y, Du G, et al. Arginine methyltransferase PRMT5 methylates and stabilizes KLF5 via decreasing its phosphorylation and ubiquitination to promote basal-like breast cancer. Cell Death Differ. 2021.

29. Guo P, Zhao KW, Dong XY, Sun X, Dong JT. Acetylation of KLF5 alters the assembly of p15 transcription factors in transforming growth factor-beta-mediated induction in epithelial cells. J Biol Chem. 2009; 284: 18184-93.

30. Guo $\mathrm{P}$, Xing $\mathrm{C}, \mathrm{Fu} X, \mathrm{He} \mathrm{D}$, Dong JT. Ras inhibits TGF- $\beta$-induced KLF5 acetylation and transcriptional complex assembly via regulating SMAD2/3 phosphorylation in epithelial cells. J Cell Biochem. 2020; 121: 2197-208.

31. Bolanos-Meade J, Reshef R, Fraser R, Fei M, Abhyankar S, Al-Kadhimi Z, et al. Three prophylaxis regimens (tacrolimus, mycophenolate mofetil, and cyclophosphamide; tacrolimus, methotrexate, and bortezomib; or tacrolimus, methotrexate, and maraviroc) versus tacrolimus and methotrexate for 
prevention of graft-versus-host disease with haemopoietic cell transplantation with reduced-intensity conditioning: a randomised phase 2 trial with a non-randomised contemporaneous control group (BMT CTN 1203). Lancet Haematol. 2019; 6: e132-e43.

32. Zhang B, Ci X, Tao R, Ni JJ, Xuan X, King JL, et al. Klf5 acetylation regulates luminal differentiation of basal progenitors in prostate development and regeneration. Nature communications. 2020; 11: 997.

33. Nagai R, Suzuki T, Aizawa K, Shindo T, Manabe I. Significance of the transcription factor KLF5 in cardiovascular remodeling. J Thromb Haemost. 2005; 3: 1569-76.

34. Tao R, Zhang B, Li Y, King JL, Tian R, Xia S, et al. HDAC-mediated deacetylation of KLF5 associates with its proteasomal degradation. Biochemical and biophysical research communications. 2018; 500: 777-82.

35. Wei $W$, Chen $W$, He N. HDAC4 induces the development of asthma by increasing Slug-upregulated CXCL12 expression through KLF5 deacetylation. J Transl Med. 2021; 19: 258.

36. Chen C, Sun X, Ran Q, Wilkinson KD, Murphy TJ, Simons JW, et al. Ubiquitin-proteasome degradation of KLF5 transcription factor in cancer and untransformed epithelial cells. Oncogene. 2005; 24: 3319-27.

37. Chen C, Sun X, Guo P, Dong XY, Sethi P, Cheng X, et al. Human Kruppel-like factor 5 is a target of the E3 ubiquitin ligase WWP1 for proteolysis in epithelial cells. J Biol Chem. 2005; 280: 41553-61.

38. Du JX, Hagos EG, Nandan MO, Bialkowska AB, Yu B, Yang VW. The E3 ubiquitin ligase SMAD ubiquitination regulatory factor 2 negatively regulates Kruppel-like factor 5 protein. J Biol Chem. 2011; 286: 40354-64.

39. Zhao D, Zheng H-Q, Zhou Z, Chen C. The Fbw7 Tumor Suppressor Targets KLF5 for Ubiquitin-Mediated Degradation and Suppresses Breast Cell Proliferation. Cancer Research. 2010; 70: 4728-38.

40. Zhao D, Zhi X, Zhou Z, Chen C. TAZ antagonizes the WWP1-mediated KLF5 degradation and promotes breast cell proliferation and tumorigenesis. Carcinogenesis. 2012; 33: 59-67.

41. Zhi X, Zhao D, Zhou Z, Liu R, Chen C. YAP promotes breast cell proliferation and survival partially through stabilizing the KLF5 transcription factor. Am J Pathol. 2012; 180: 2452-61.

42. Ge F, Chen W, Qin J, Zhou Z, Liu R, Liu L, et al. Ataxin-3 like (ATXN3L), a member of the Josephin family of deubiquitinating enzymes, promotes breast cancer proliferation by deubiquitinating Kruppel-like factor 5 (KLF5). Oncotarget. 2015; 6: 21369-78.

43. Wu Y, Qin J, Li F, Yang C, Li Z, Zhou Z, et al. USP3 promotes breast cancer cell proliferation by deubiquitinating KLF5. J Biol Chem. 2019; 294: 17837-47.

44. Giandomenico V, Simonsson M, Gronroos E, Ericsson J. Coactivator-dependent acetylation stabilizes members of the SREBP family of transcription factors. Mol Cell Biol. 2003; 23: 2587-99.

45. Simonsson $\mathrm{M}$, Heldin $\mathrm{CH}$, Ericsson J, Gronroos E. The balance between acetylation and deacetylation controls Smad7 stability. J Biol Chem. 2005; 280: 21797-803.

46. Jeong JW, Bae MK, Ahn MY, Kim SH, Sohn TK, Bae MH, et al. Regulation and destabilization of HIF-1alpha by ARD1-mediated acetylation. Cell. 2002; 111: 709-20.

47. Leduc C, Claverie P, Eymin B, Col E, Khochbin S, Brambilla E, et al. p14ARF promotes RB accumulation through inhibition of its Tip60-dependent acetylation. Oncogene. 2006; 25: 4147-54.

48. Guo P, Dong XY, Zhang X, Zhao KW, Sun X, Li Q, et al. Pro-proliferative factor KLF5 becomes anti-proliferative in epithelial homeostasis upon signaling-mediated modification. J Biol Chem. 2009; 284: 6071-8.

49. Damaskos C, Garmpi A, Nikolettos K, Vavourakis M, Diamantis E, Patsouras A, et al. Triple-Negative Breast Cancer: The Progress of Targeted Therapies and Future Tendencies. Anticancer Res. 2019; 39: 5285-96.

50. Tao R, Zhang BT, Li YX, King JL, Tian RY, Xia SY, et al. HDAC-mediated deacetylation of KLF5 associates with its proteasomal degradation. Biochem Bioph Res Co. 2018; 500: 777-82

51. Oin J, Zhou Z, Chen W, Wang C, Zhang H, Ge G, et al. BAP1 promotes breast cancer cell proliferation and metastasis by deubiquitinating KLF5. Nature Communications. 2015; 6: 8471

52. Zhao D, Zheng HQ, Zhou Z, Chen $\mathrm{C}$. The Fbw7 tumor suppressor targets KLF5 for ubiquitin-mediated degradation and suppresses breast cell proliferation. Cancer Res. 2010; 70: 4728-38.

53. Lin CL, Tsai ML, Lin CY, Hsu KW, Hsieh WS, Chi WM, et al. HDAC1 and HDAC2 Double Knockout Triggers Cell Apoptosis in Advanced Thyroid Cancer. Int J Mol Sci. 2019; 20.

54. Matsumura T, Suzuki T, Aizawa K, Munemasa Y, Muto S, Horikoshi M, et al. The deacetylase HDAC1 negatively regulates the cardiovascular transcription factor Kruppel-like factor 5 through direct interaction. J Biol Chem. 2005; 280: 12123-9.

55. Zhang B, Li Y, Wu O, Xie L, Barwick B, Fu C, et al. Acetylation of KLF5 maintains EMT and tumorigenicity to cause chemoresistant bone metastasis in prostate cancer. Nat Commun. 2021; 12: 1714.

56. Hernandez-Hernandez A, Ray P, Litos G, Ciro M, Ottolenghi S, Beug H, et al. Acetylation and MAPK phosphorylation cooperate to regulate the degradation of active GATA-1. EMBO J. 2006; 25: 3264-74.

57. Jiang YZ, Ma D, Suo C, Shi J, Xue M, Hu X, et al. Genomic and Transcriptomic Landscape of Triple-Negative Breast Cancers: Subtypes and Treatment Strategies. Cancer Cell. 2019; 35: 428-40 e5.
58. Zhao C, Li Y, Oiu W, He F, Zhang W, Zhao D, et al. C5a induces A549 cell proliferation of non-small cell lung cancer via GDF15 gene activation mediated by GCN5-dependent KLF5 acetylation. Oncogene. 2018; 37: 4821-37.

59. Miyamoto S, Suzuki T, Muto S, Aizawa K, Kimura A, Mizuno Y, et al. Positive and negative regulation of the cardiovascular transcription factor KLF5 by p300 and the oncogenic regulator SET through interaction and acetylation on the DNA-binding domain. Mol Cell Biol. 2003; 23: 8528-41.

60. Jia J, Zhang HB, Shi Q, Yang C, Ma JB, Jin B, et al. KLF5 downregulation desensitizes castration-resistant prostate cancer cells to docetaxel by increasing BECN1 expression and inducing cell autophagy. Theranostics. 2019; 9: 5464-77.

61. Jiang Z, Li W, Hu X, Zhang Q, Sun T, Cui S, et al. Tucidinostat plus exemestane for postmenopausal patients with advanced, hormone receptor-positive breast cancer (ACE): a randomised, double-blind, placebo-controlled, phase 3 trial. Lancet Oncol. 2019; 20: 806-15.

62. Ramaswamy B, Fiskus W, Cohen B, Pellegrino C, Hershman DL, Chuang E, et al. Phase I-II study of vorinostat plus paclitaxel and bevacizumab in metastatic breast cancer: evidence for vorinostat-induced tubulin acetylation and Hsp90 inhibition in vivo. Breast Cancer Res Treat. 2012; 132: 1063-72.

63. Connolly RM, Fackler MJ, Zhang Z, Zhou XC, Goetz MP, Boughey JC, et al. Tumor and serum DNA methylation in women receiving preoperative chemotherapy with or without vorinostat in TBCRC008. Breast Cancer Res Treat. 2018; 167: 107-16.

64. Connolly RM, Leal JP, Goetz MP, Zhang Z, Zhou XC, Jacobs LK, et al. TBCRC 008: early change in 18F-FDG uptake on PET predicts response to preoperative systemic therapy in human epidermal growth factor receptor 2-negative primary operable breast cancer. J Nucl Med. 2015; 56: 31-7. 